\title{
Ontogenetic Aspect of Steroidogenesis by Gonads and Adrenals of Ducks and Its Role on Sex Differentiation
}

\author{
Yuichi Tanabe*
}

\begin{abstract}
Plasma testosterone, estradiol, corticosterone and cortisol levels together with gonadal testosterone and estradiol concentrations were determined by radioimmunoassay in the embryonic and post-embryonic Domestic Duck, Anas platyrhynchos var. domestica. The plasma testosterone and estradiol concentrations were higher in the female than in the male embryo, whereas no sex-difference was observed either in plasma corticosterone or cortisol. Both the ovarian estradiol and testosterone concentrations in the embryo were much higher in the testicular concentrations. The plasma corticosterone or cortisol concentration reached a peak one day after hatching, and decreased thereafter in both sexes. These results indicate that the ovary of embryonic duck is more active in secreting the sex steroid hormones than the embryonic testes, and suggest that the original sex of the duck (probably of all avian species) should be male (ZZ), and estrogenic hormones secreted from the embryonic ovary has an important role in sexual development (feminizing) of the female in the duck.
\end{abstract}

\section{Introduction}

Many works have been done on ontogenetic steroidogenesis in mammals (Rigaudière 1977, Geroge et al. 1978, Wilson 1978). Whereas in birds some works have been reported only in the chicken (Woods et al. 1975, Woods \& Brazzill 1981, Guichard et al. 1977, 1979, Tanabe et al. 1979). Recently, it was found that H-Y antigen plays a pivotal role in the primary (gonadal) sex determination of mammals, and its regulatory gene exsists on Y chromosome of XY male cells in mammals, but it exists in ZW female cells (possibly on W chromosome) in avian species (Wachtel et al. 1975 a b, Ohno 1979, Ohno \& Matsunaga 1981, Müller et al. 1979, Zaborski et al. 1980).

The present work was undertaken to ellucidate the relationship between ontogenetic steroidogenesis and sex differentiation in Domestic Ducks (Anas platyrhyncos).

\section{Materials and Methods}

The duck and ducklings were obtained from Osaka-Kairyo Breed, bred from a hybrid between Japanese native and Pekin duck. The eggs were incubated in an incubator at $38^{\circ} \mathrm{C}$ and $70 \%$ relative humidity, and hatched on the 27 th day of incubation. Blood samples were collected with a heparinized syringe by heart puncture from 8 embryos of both sexes at 15, 19, 23 and 26 days of incubation, from 8 ducklings of both sexes at one and three days, and from 5 ducklings of both sexes at 7 and 14 days after hatching. The blood samples were centrifuged for separation of plasma.

One tenth ml plasma was used for the determination of testosterone, corticosterone

\footnotetext{
* Department of Poultry and Animal Sciences, Gifu University, Yanagido, Gifu 501-11, Japan.
} 
and cortisol, and $0.2 \mathrm{ml}$ plasma for estradiol. After killing the embryos or ducklings, left ovary or testis was removed. Ethyl ether extractions were repeated three times for the steroid hormones.

The radioimmunoassay without chromatography was carried out for estradiol, testosterone, corticosterone and cortisol (Shodono et al. 1975, Tanabe et al. 1979).

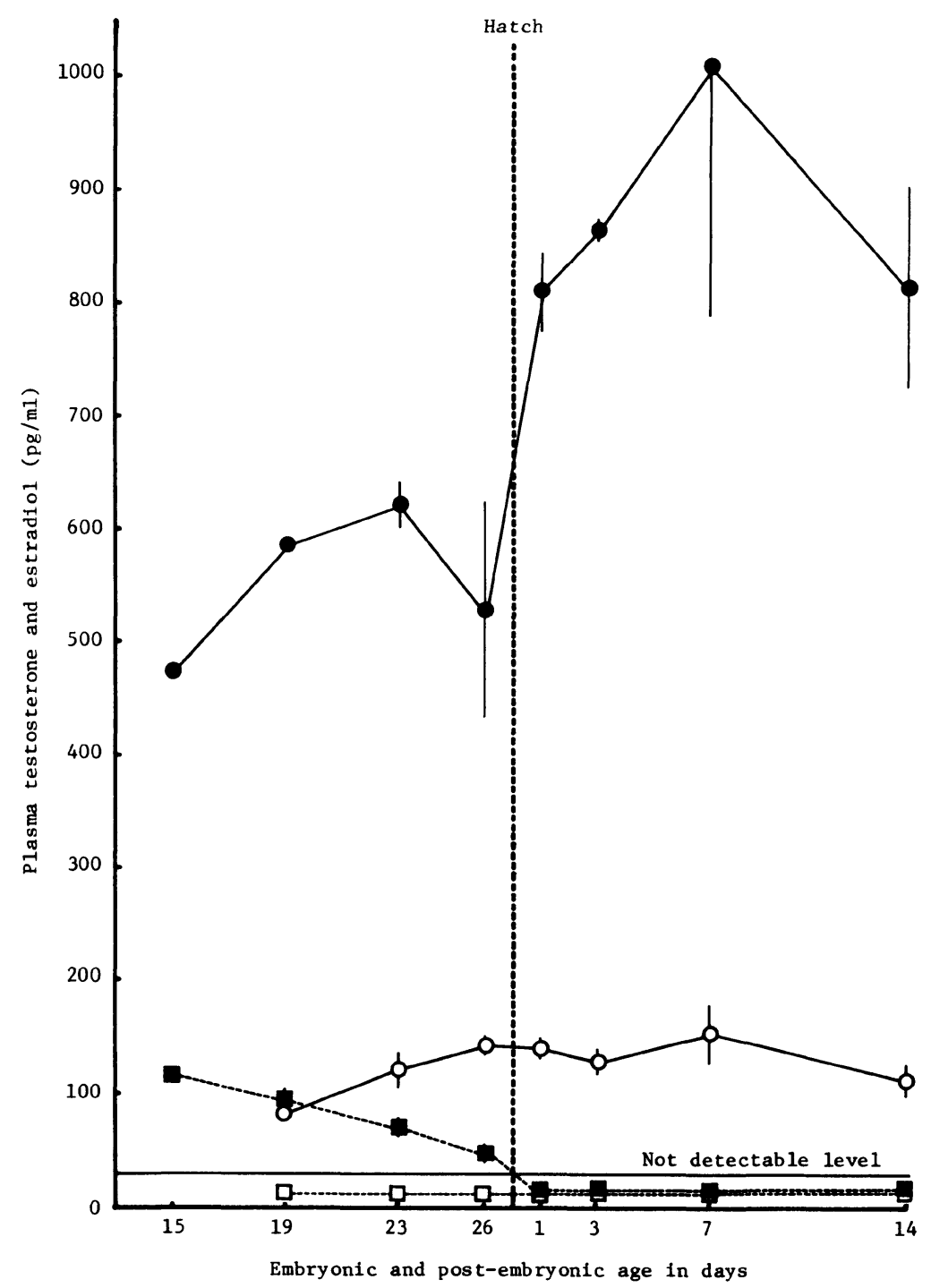

Fig. 1. Plasma testosterone (male $\bigcirc-\bigcirc$, female $-O$ ) and estradiol (male $\square-\square$, female - 1 ) concentrations of embryonic and post-embryonic ducks. Each point and vertical line represent mean of 8 embryos or 5-8 ducklings and SEM, respectively. 


\section{Results}

Plasma testosterone and estradiol concentrations of 15-, 19-, 23-, and 26-day-old embryos and 1-, 3-, 7-, and 14-day-old ducklings are illustrated in Fig. 1. Plasma testosterone levels were much higher in the female than the male throughout the embryonic and post-embryonic ages. Plasma estradiol levels were higher in the female than in the male embryo, whereas no significant amount of estradiol was detected in post-embryonic ducks of both sexes.

Ovarian and testicular concentrations of estradiol and testosterone of ducks at the embryonic and post-embryonic ducks are illustrated in Fig. 2. Both estradiol and testosterone concentrations were much higher in the ovary than in the testis. The

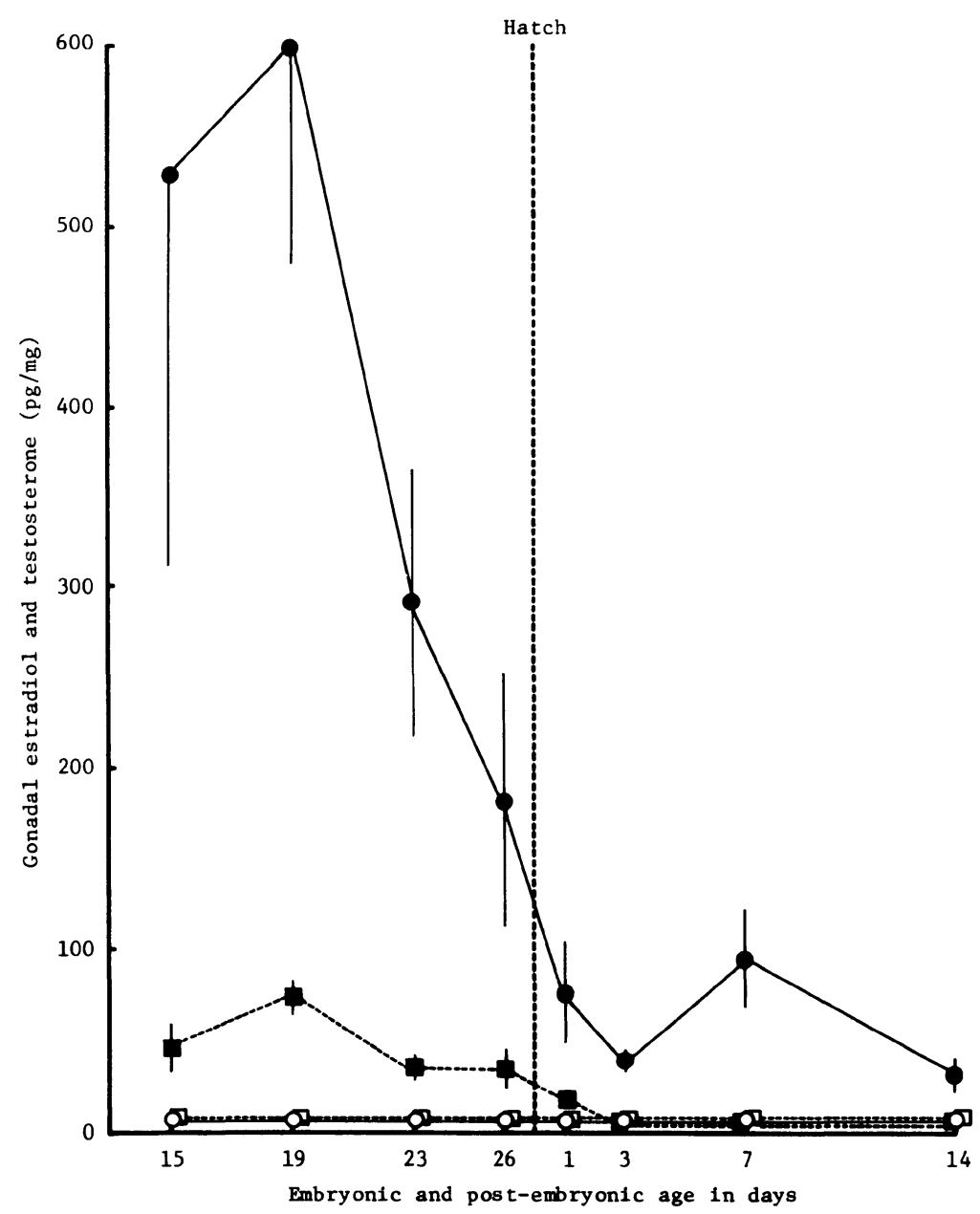

Fig. 2. Concentrations of testosterone in testis $(\square-\square)$ or in ovary ( $\square-\square$ ) and estradiol in testis $(\mathrm{O}-\mathrm{O})$ or in ovary $(-\mathrm{O})$. Each spot and vertical line represent mean of 8 embryos or 5-8 ducklings and SEM, respectively. 
ovarian concentrations of both hormones decreased after hatching. The levels of estradiol in the ovary were still higher than the testicular concentrations even 14-day-old ducklings. A similar sex-difference in the gonadal progesterone concentration was also observed (Tanabe et al. 1983).

Plasma corticosterone and cortisol levels in embryonic and post-embryonic ducks are illustrated in Fig. 3. Plasma corticosterone levels were low in 15-, 19- and 23-day-old embryos of both sexes, increased rapidly in 26-day-old embryos, reached a peak around the hatching time, and decreased rapidly thereafter. Similar changes in the plasma cortisol levels were observed although the cortisol levels were much less than the corticosterone cencentration. No significant sex-difference was observed either in the plasma corticosterone or cortisol levels.

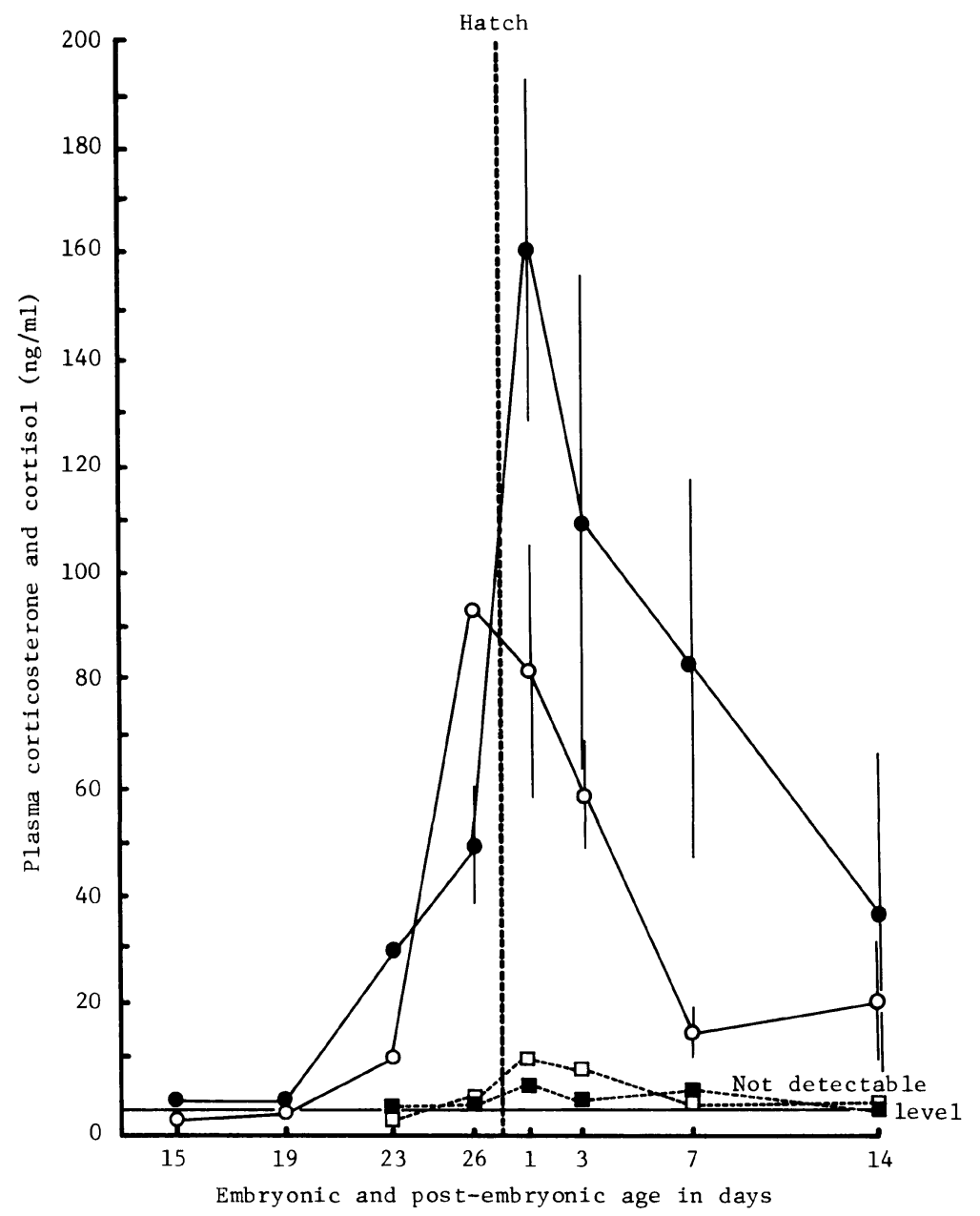

Fig. 3. Concentrations of plasma corticosterone (male $\bigcirc-\bigcirc$, female - - ) and plasma cortisol (male $\square-\square$, female $\square-\square$ ). Each spot and vertical line represent mean of 8 embryos or 5-8 ducklings and SEM, respectively. 


\section{Discussion}

The present study indicates that duck embryonic and postembryonic ovaries are highly active in the production and secretion of testosterone and estradiol as compared with embryonic and postembryonic testes. Similar observations have been made in the embryonic chickens (Guichard et al. 1977, 1979, Tanabe et al. 1979). This is in contrast with the case of mammalian embryo, in which embryonic testes are much more active than embryonic ovaries (Rigaudière 1977, George et al. 1978, Wilson 1978).

In rabits, gonadectomy in male or female embryo prior to the onst of phenotypic differentiation results in the development of the female phenotype (Jost 1972). On the contrary, in the avian species, the male secondaries such as the development of phallus and the laryngeal extension in ducks (Wolff \& Wolff 1949) and the male type plumage in the chicken (Morgan 1920) is known to appear in castrated females.

It is to be noted that application of sex-steroid in the species with male heterozygoty $(X X / X Y)$ are apt to induce musculinization of the female $(X X)$, whereas in the case of female heterozygoty $(\mathrm{ZW} / \mathrm{ZZ})$, similar treatments are tended to cause feminization of the male (ZZ), in amphibian, avian and mammalian species (Witschi 1967, Wolff \& Wolff 1949). Furthermore, there exists abundant evidence indicative that in mmmals $X Y$ male cells disseminate testis-organizing $\mathrm{H}-\mathrm{Y}$ antigen, while in avian species $\mathrm{ZW}$ female cells disseminate ovary-organizing H-Y antigen (Wachtel et al. 1975a b, Ohno 1979, Ohno and Matsunaga 1981). Recently, Müller et al. (1978) reported the induction of H-Y antigen by estradiol benzoate in the gonads of male (ZZ) chicken embryo, thus ruling out a W chromosomal location of the structural gene of the antigen. This observation, along with the disappearance of $\mathrm{H}-\mathrm{Y}$ antigen during the transformation of right gonad into testes in ovariectomized chicks (Zaborski et al. 1980), suggests that $\mathrm{H}-\mathrm{Y}$ antigen is rather a differentiation factor than a determining factor in the organization of the ovary in the avian species, and that the expression of the antigen in avian species is regulated by estrogen. Taken all together, these observations on $\mathrm{H}-\mathrm{Y}$ antigen indicate that the sex of the avian species is basically (inherently) male with homozygoty of sex chromosone $(\mathrm{ZZ})$, and that the estrogens secreted from the embryonic ovary plays a very important role in sex differentiation and sexual development (feminizing) of the female. Furthermore, the major sex-determining (regulatory) gene is present on $\mathrm{W}$ chromosome, and the structural gene for $\mathrm{H}-\mathrm{Y}$ antigen like-substance is present on autosomes; the avian female is the induced sex in contrast to mammals.

In the present study, plasma corticosterone levels reached a peak around the hatching time in both sexes of ducks. Although the plasma levels of cortisol were much less than those of corticosterone, they showed a similar change during embryonic and postembryonic (until 14 days of age) ducks as in the corticosterone concentrations. Both the cortisosterone and cortisol levels decreased after hatching. It is likely that higher levels of corticosteroids are needed for newly hatched ducklings to adapt to a new environment after hatching. 


\section{Acknowledgements}

I wish to thank Dr. Takao Nakamura and Mr. Takashi Yano, Gifu University for their cooperation in this study.

\section{References}

George, F. W., Wilewich, L. \& Wilson, J. D. 1978. Oestrogen content of the embryonic rabit ovary. Nature (London) 274: 172-173.

Guichard, A., Cedard, L., Mignot, Th.-M., Scheib, D. \& Haffen, K. 1977. Radioimmunoassay of steroids produced by cultured chick embryonic gonads: Difference according to age, sex, and size. Gen. Com. Endocrinol. 32: 255-265.

Guichard, A., Cedard, L., Mignot, Th.-M., Scheib, D. \& Haffen, K. 1979. Radioimmunoassay of steroids by chick embryo gonads cultured in the presence of some exogenous steroid precursors. Gen. Comp. Endocrinol. 39: 9-19.

Hattori, M. \& Wakabyashi, K. 1979. Isoelectric forcusing and filtration studies on the heterogeneity of avian pituitary luteinizing hormone. Gen. Comp. Endocrinol. 39: 215-221.

Jost, A. 1972. A new look at the mechanism controlling sex differentiation in mammals. Johns Hopkins Med. J. 130: $38-53$.

Morgan, T. H. 1920. The effects of castration of hen feathered Campines. Biol. Bull. 39: 231-247.

Müller, U., Zenzes, M. T., Wolf, U., Engle, W. \& Weniger, J.P. 1979. Appearance of H-W (H-Y) antigen in the gonads of oestradiol sex-reversed male chicken embryos. Nature (London) 280: 142-144.

Ohno, S. 1979. Major Sex-determining Genes. Springer-Verlag, Berlin. 140 pp.

Ohno, S \& Matsunaga, T. 1981. The role of H-Y plasma membrane antigen in the evolution of the chromosomal sex determining mechanism. In Levels of Genetic control in Development. Subtelmy, S. \& Abbott, U. K. eds., Alan R. Liss, Inc., N. Y. 225-246 pp.

Rigaudière, N. 1977. Évolution des teneurs testostérone et dihydrotestostérone dans le plasma, le testicule et l'ovaire chez le cobaye au cours de la vie foetale. C. R. Acad. Sc. (Paris) 285 D: 989-992.

Shodono, M., Nakamura, T., Tanabe, Y. \& Wakabayashi, K. 1975. Simultaneous determinations of oestradiol-17 $\beta$, progesterone and luteinizing hormone in the plasma during the ovulatory cycle of the hen. Acta. Endocinol. 78: 565-573.

Tanabe, Y., Nakamura, T., Fujioka, K. \& Doi, O. 1979. Production and secretion of sex steroid hormone by the testes, the ovary and the adrenal glands of embryonic and young chickens (Gallus domesticus). Gen. Comp. Endocrinol. 39: 26-33.

Tanabe, Y., Yano, T. \& Nakamura, T. 1983. Steroid hormone synthesis and secretion by testes, ovary, and adrenals of embryonic and postembyonic ducks. Gen. Comp. Endocrinol. 49: in press.

Wachtel, S. S., Ohno, S., Koo, G. C. \& Boyse, E. A. 1975a. Possible role of H-Y antigen in primary sex determination. Nature (London) 257: 235-256.

Wachtel, S. S., Koo, G. C. \& Boyse, E. A. 1975b. Evolutionary conservation of H-Y (male) antigen. Nature (London) 254: 270-272.

Wilson, J. D. 1978. Sexual differentiation. Ann. Rev. Physiol. 40: 279-306.

Witschi, E. 1967. Biochemistry of sex differentiation in vertebrate embryos. In The Biochemistry of Animal Development. Vol. II. Weber, R. ed., 193-225 pp. Academic Press, N. Y.

Wolff, Et. \& Wolff, Em. 1949. Application de la méthode de castration à l'embryon de Canard: Sur deux tests de l'activité précoce des gonades embryonnaires, la syrinx et le tubercule genital. C. R. Soc. Biol. 143: 529-531.

Woods, J. E., Simpson, R. M. \& Moore, P. L. 1975. Plasma testosterone levels in the chick embryo. Gen. Comp. Endocrinol. 27: 543-547.

Woods, J. E. \& Brazzill, D. M. 1981. Plasma 17 $\beta$-estradiol levels in the chick embryo. Gen. Comp. Endocrinol. 44: 37-43.

Zaborski, P., Guichard, A. \& Reyss-Brion, M. 1980. H-Y antigen in ovariectomized chicks: Disappearance of its extression during the transformation of the right gonad into a testis. Biol. Cellulaire 39: 291-294. 